ÉGYPTE

monde arabe

\section{Égypte/Monde arabe}

9 | 1992

Vers une économie libérale?

\title{
Le programme de stabilisation et d'ajustement structurel de l'économie égyptienne
}

\section{Louis Blin}

\section{(2) OpenEdition}

\section{Journals}

Édition électronique

URL : https://journals.openedition.org/ema/1211

DOI : 10.4000/ema.1211

ISSN : 2090-7273

Éditeur

CEDEJ - Centre d'études et de documentation économiques juridiques et sociales

Édition imprimée

Date de publication : 31 mars 1992

Pagination : 13-46

ISSN : 1110-5097

\section{Référence électronique}

Louis Blin, «Le programme de stabilisation et d'ajustement structurel de l'économie égyptienne »,

Égypte/Monde arabe [En ligne], 9 | 1992, mis en ligne le 08 juillet 2008, consulté le 07 juillet 2022. URL: http://journals.openedition.org/ema/1211 ; DOI : https://doi.org/10.4000/ema.1211

Ce document a été généré automatiquement le 7 juillet 2022.

Tous droits réservés 


\title{
Le programme de stabilisation et d'ajustement structurel de l'économie égyptienne
}

\author{
Louis Blin
}

1 Cet article a pour objet d'exposer le programme de stabilisation et d'ajustement structurel de l'économie de l'Égypte et d'évaluer sa mise en oeuvre à la fin mars 1992. Il ne s'agit donc pas d'une analyse de sa pertinence ou des causes qui ont amené les autorités de ce pays à commencer à appliquer certaines de ses dispositions de manière autonome dès mars 1990, puis de façon plus globale et contraignante en coopération avec les institutions internationales de financement à partir de mai 1991.

2 Nous examinerons successivement le soutien international à sa mise en place, la façon dont le gouvernement égyptien l'a présenté au pays et son contenu. Nous distinguerons à cet effet la stabilisation de l'ajustement, bien qu'il s'agisse d'un tout indissociable. Nous exposerons par la suite le projet de Fonds social qui accompagne ce programme et les prévisions macro-économiques qui en découlent.

\section{Un programme fortement soutenu de l'extérieur}

3 L'Égypte s'est engagée en 1991 dans un programme de stabilisation et d'ajustement structurel de son économie, dont les deux premières années seront financées par environ 1,5 milliard $\$$ de crédits d'organismes internationaux :

4 - Le programme de stabilisation bénéficie d'un crédit de confirmation (stand by) de 278 millions de DTS (372 millions \$ au moment de la signature) en deux tranches égales accordé le 17 mai 1991 par le Fonds Monétaire International (FMI) pour une période de dix-huit mois.

5 - Le programme d'ajustement est financé par la Banque Mondiale (prêt à l'ajustement structurel de 300 millions \$ en deux tranches égales approuvé le 24 juin et signé le 22 novembre 1991), la Banque Africaine de Développement (100 millions d'unités de 
compte en deux tranches, soit 140 millions $\$$ au moment de l'approbation du prêt le 24 septembre 1991) et la Communauté Economique Européenne (60 100 millions d'écus, soit 79-132 millions $\$$ devant financer la deuxième phase du programme, don non encore signé).

6 - En complément, un Fonds social d'aide à l'ajustement structurel a été mis sur pied sous l'égide de l'Association Internationale de Développement (AID), filiale de la Banque Mondiale intervenant en faveur des pays les plus pauvres, qui n'opérait plus en Égypte depuis 1981. L'AID a attribué le 27 septembre 1991 un prêt de 105 millions de DTS (140 millions \$), auxquels s'ajoutaient fin janvier 1992180 millions \$ de prêts et 289,45 millions $\$$ de dons (dont 55 millions $\$$ d'engagements) internationaux, soit au total 609,45 millions $\$$.

7 - Le FMI et la Banque Mondiale jouant le rôle de catalyseurs de l'aide internationale, l'adoption du programme de stabilisation et d'ajustement structurel et la signature des prêts le finançant a permis la conclusion par l'Égypte d'autres accords financiers d'un montant global de 16,2 milliards $\$$, qui constituent des soutiens indirects à sa réussite :

8 - Accord de réduction de moitié et de rééchelonnement du solde de la dette publique, signé le 25 mai 1991, effaçant 18,2 milliards \$ de dettes (en outre, les pays du Golfe et les États-Unis avaient remis quelque 14 milliards $\$$ de dettes égyptiennes avant la signature de l'accord avec le FMI).

9 - Accord conclu le 10 juillet suivant avec le groupe consultatif des bailleurs de fonds de l'Égypte prévoyant l'attribution de 8 milliards $\$$ de prêts et dons jusqu'à la fin de l'année fiscale 1992-93' ${ }^{1}$, comprenant leurs contributions au Fonds social. La prochaine réunion du groupe consultatif est prévue pour novembre 1992.

10 Le montant total de l'aide internationale obtenue par l'Égypte grâce à son engagement à assainir son économie s'élève donc à 17,25 milliards \$, soit environ $300000 \$$ par Égyptien. S'ajouteront à cette somme des transferts privés induits par l'amélioration des perspectives économiques du pays, au montant non prévisible mais chiffrable en milliards de dollars, plus éventuellement d'autres prêts non encore signés destinés à financer des réformes ultérieures, l'ajustement ne pouvant être achevé en deux ans.

11 La Banque Mondiale devrait notamment continuer à financer l'ajustement structurel si ce dernier se déroule conformément à ses voeux. Le prêt à l'ajustement structurel approuvé en juin 1991 devrait en effet être suivi par un prêt complémentaire. Les prêts envisagés par le groupe de la Banque Mondiale durant les trois exercices allant de 1991-92 à 1993-94 atteignent 1,5 milliard \$, contre 311,5 millions \$ seulement pendant les cinq années 1986-90 et des prêts cumulés de 4,2 milliards $\$$ à la fin de 1991, dont 2,9 milliards $\$$ décaissés.

12 Les versements de prêts et les remises de dettes interviendront en plusieurs tranches, étant conditionnés au respect des mesures définies avec le FMI et la Banque Mondiale et régulièrement contrôlées par leurs missions d'évaluation comme par les bureaux que ces organismes ont ouverts au Caire. Tout retard de l'Égypte dans leur application remet en cause l'ensemble des versements. L'exceptionnel état de grâce international dont bénéficie l'Égypte pour deux ans n'exclut en effet pas les contrôles, la méfiance à l'égard des capacités politiques à libéraliser l'économie grâce à ce programme étant profonde. 


\section{Une politique difficilement assumée}

13 Le programme de stabilisation et d'ajustement structurel de l'économie égyptienne n'a jamais été rendu public de façon détaillée par le gouvernement. Les autorités ont exposé certaines des mesures auxquelles elles se sont engagées vis-à-vis de leurs créditeurs internationaux, généralement lorsqu'elles nécessitaient un passage devant le Parlement et pas toujours de manière claire, appliquant les autres sans annonce préalable ${ }^{2}$.

14 Le président Moubarak a annoncé les grandes lignes de ce programme, baptisé " programme des 1000 jours » en référence à sa durée approximative attendue, dans une allocution prononcée le 1er mai 1990. Les autorités égyptiennes ont ensuite repris régulièrement l'argumentation développée dans ce discours, dans lequel M. Moubarak qualifiait le FMI de "Fonds de la Misère Internationale » ${ }^{3}$ et estimait: "Il n'y a pas d'autre issue, nous devons supporter cela ", se posant donc en victime des pressions extérieures.

15 Le principe d'un tel programme avait ainsi été adopté par les autorités bien avant la signature avec le FMI, son maître d'oeuvre habituel, et certaines de ses dispositions ont commencé à être mises en oeuvre dès mars 1990. La lenteur des négociations avec le Fonds (environ trois ans) est en premier lieu due au fait que ce dernier avait été rendu méfiant par le précédent malheureux de l'accord de 1987, non respecté par les Égyptiens. En négociateurs éprouvés, ces derniers n'ont cependant pas hésité à utiliser tous leurs atouts pour obtenir les meilleures conditions possibles, laissant également percer ainsi leur manque d'assurance à l'égard de leur capacité de mener à bien ce programme. Les autorités ont ainsi choisi de présenter en public une approche négative des réformes mises en oeuvre, ne soulignant pas que le statu quo aurait été autrement plus difficile à supporter, ce qui aurait pourtant été aisé à démontrer au regard de la rapide dégradation de la situation économique du pays depuis 1987. Cette dernière option aurait cependant mis en relief les tares de la gestion menée par une équipe gouvernementale inchangée depuis lors. Le président a en effet renouvelé sa confiance à ses ministres économiques lors du remaniement de mai 1991 ayant suivi la signature de l'accord avec le FMI, déjouant la plupart des pronostics.

16 À l'instar d'autres gouvernements et en ligne avec la discrétion des institutions financières internationales, les autorités égyptiennes préfèrent l'ambiguïté au débat public. Elles considèrent sans doute que l'impopularité présupposée de ce programme peut entraver son application s'il est publié et que certains engagements prêtent le flanc aux critiques. Les campagnes gouvernementales de promotion de sa politique économique sont concentrées sur certains points variant en fonction de la conjoncture et des interlocuteurs. C'est aussi une façon de ménager d'éventuelles adaptations pragmatiques en cours de route, vu que l'expérience des pays ayant déjà appliqué ce type de programme montre que son application relève d'une négociation continue entre le gouvernement et ses bailleurs de fonds étrangers, arbitrée de fait par la réponse du pays aux mesures mises en oeuvre.

17 L'attitude du gouvernement a conduit au résultat paradoxal qu'il a quasiment passé sous silence les appréciations remarquables de sa politique économique émises par le FMI et la Banque mondiale en mars 1992. Cette difficulté non avouée des autorités égyptiennes à assumer publiquement leur politique économique, contrastant par exemple avec les expériences menées simultanément dans les ex-pays socialistes 
d'Europe, s'explique par les rapports entretenus par la société égyptienne avec son État. Son analyse sortirait du cadre de cet article.

\section{Le programme de stabilisation}

18 Le gouvernement égyptien a préparé dès 1989 un "programme élargi d'ajustement structurel et de réforme économique " qui devait être mis en oeuvre pendant une période de six ans allant de l'année fiscale 1989-90 à 1994-95. Il a introduit dès mars 1990 des réformes allant dans le sens des mesures préconisées par le FMI, tout en négociant avec ce dernier le soutien international : indispensable à leur réussite.

19 Le programme de stabilisation de l'économie égyptienne, préparé et mis en oeuvre en coopération avec le FMI, vise à restaurer l'équilibre macro-économique du pays et à y réduire l'inflation. Les quatre grands domaines concernés par ce programme sont la fiscalité, la politique monétaire, le marché des changes et le système bancaire.

\section{Fiscalité}

20 La réforme fiscale a pour objet d'assainir les finances publiques et de permettre de réduire le rôle économique de l'État. Elle consiste essentiellement à ramener le ratio emprunts du secteur public/produit intérieur brut de $21,9 \%$ durant l'année fiscale 1990-91 à 6,6 \% deux ans plus tard, pour tendre vers $1,5 \%$ en 1995-96, grâce à une modification du financement et à une réduction du déficit budgétaire. Ce dernier devrait chuter de $21,9 \%$ du PIB en 1990-91 à 10,2\% l'année suivante et tendre vers 1,5 \% en 1995-96, grâce à la hausse des recettes budgétaires et à la baisse des dépenses, relativement au PIB.

21 Une hausse des recettes du budget de l'État, de $26 \%$ du PIB en 1990-91 à $37 \%$ en 1995-96 (voir tableau 1 p. suivante), est attendue de l'augmentation des recettes fiscales et non-fiscales.

L'augmentation des recettes fiscales, dès la fin de l'année 1990-91, provient de la mise en place (effectuée à partir du 28 avril 1991) d'une "taxe générale sur les ventes ", d'une augmentation des droits de douane, des taxes sur les cigarettes et d'une hausse des tarifs des services publics. Ces recettes passeraient de 18,5\% du PIB en 1990-91 à 23,9\% en 1995-96. Une réforme de la fiscalité sur les revenus et sur les entreprises, si complexe que les experts internationaux ont du mal à l'évaluer, est prévue dans le cadre de la deuxième phase de l'ajustement structurel.

Les recettes non-fiscales progresseraient quant à elles de 7,6\% du PIB en 1990-91 à $13 \%$ en 1995-96, principalement grâce à la hausse des prix des produits énergétiques (voir ci-après, " politique tarifaire »).

24 La baisse des dépenses budgétaires, de 51,5\% du PIB en 1990-91 à 42,1 \% en 1995-96, proviendra principalement de la chute des dépenses d'investissement, de 19,1\% du PIB en 1990-91 à $8,2 \%$ en 1995-96, ainsi que de la réduction des subventions à la consommation, de 5,1 milliards LE en 1990-91 à 3,1 milliards LE en 1995-96.

La modification du mode de financement du déficit budgétaire repose sur une réduction en termes réels du recours au système de sécurité sociale et aux crédits de la Banque Centrale et son remplacement par l'émission de bons du Trésor. Le financement bancaire du déficit budgétaire, qui représentait ces dernières années 
$42,5 \%$ du total en moyenne, est tombé à $3 \%$ du besoin de financement en 1991-92 et devrait disparaître en 1992-93.

Tableau 1 : Évolution des finances publiques (\% du PIB)

\begin{tabular}{|l|r|r|r|r|r|r|}
\hline & 1991 & 1992 & 1993 & 1994 & 1995 & 1996 \\
\hline Recettes totales & 29,56 & 36,88 & 36,69 & 38,27 & 39,29 & 39,96 \\
- Budget de rElat & 26,12 & 33,36 & 33,62 & 35,20 & 36,22 & 36,93 \\
- Receltes tiscales & 18,48 & 23,77 & 23,12 & 23,65 & 23,91 & 23,95 \\
- Recettes non fiscales & 7,63 & 9,59 & 10,51 & 11,55 & 12,32 & 12,98 \\
- Budget des coll. locales & 1,18 & 1,10 & 1,00 & 1,00 & 1,00 & 0,98 \\
- Services publics & 0,70 & 0,65 & 0,59 & 0,59 & 0,59 & 0,58 \\
- Autolinancement des & & & & & & \\
investissements & 1,56 & 1,78 & 1,48 & 1,47 & 1,47 & 1,46 \\
\hline Dépenses totales & 51,47 & 47,12 & 46,15 & 44,65 & 43,45 & 42,08 \\
- Dépenses de & & & & & & \\
lonctionnement & 32,40 & 33,80 & 35,92 & 35,48 & 34,75 & 33,86 \\
- Dépenses & & & & & & \\
d'investissement & 19,06 & 13,32 & 10,22 & 9,17 & 8,70 & 8,22 \\
& & & & & & \\
Défićt avant ajustement & 21,91 & 10,24 & 9,45 & 6,38 & 4,16 & 2,12 \\
Ajustement requis & 0,00 & 0,00 & 2,88 & 0,96 & 0,83 & 0,60 \\
Déficit après ajustement & 21,91 & 10,24 & 6,58 & 5,42 & 3,33 & 1,52 \\
\hline Financement du déficit & & & & & & \\
- Financement extérieur & 15,90 & 4,91 & 3,24 & 3,77 & 2,42 & 1,52 \\
net & & & & & & \\
- Financement intérieur & 6,01 & 5,33 & 3,33 & 1,65 & 0,91 & 0,00 \\
• Non bancaire & 4,20 & 4,47 & 3,33 & 1,65 & 0,91 & 0,00 \\
• Bancaire & 1,81 & 0,87 & 0,00 & 0,00 & 0,00 & 0,00 \\
& & & & & & \\
\hline
\end{tabular}

- Y compris investissements des entreprises publiques el dépenses đajustement structurel. N.B. 1991 signifie année financière 1990-91 et ainsi de suite.

Source : Banque Mondiale, juin 1991.

\section{Politique monétaire}

La nouvelle politique monétaire consiste en premier lieu à :

1. Émettre des bons du Trésor (mesure engagée à un rythme hebdomadaire le 2 janvier $1991^{4}$ afin de financer le déficit budgétaire de façon non inflationniste, d'éponger l'excès de liquidités et d'orienter les taux d'intérêt, jetant les bases d'un marché monétaire.

2. Libérer les taux débiteurs et créditeurs des banques (mesure effective le 3 janvier 1991), ce qui permet aux taux d'adjudication des bons du Trésor de devenir le taux directeur du système. Ceci devrait avoir pour conséquence de faire passer à terme les taux au-dessus de l'inflation, dissuadant de la sorte les opérateurs de s'endetter et mettant ainsi fin à la nécessité d'encadrer le crédit afin de lutter contre l'inflation résultante.

L'émission des bons du Trésor a rencontré un succès certain avec un montant cumulé de 10,2 milliards LE et un encours de 6,5 milliards LE environ à la mi-novembre 1991. Leur taux d'intérêt était suffisamment élevé à partir du troisième trimestre pour que leur souscription soit plus rémunératrice qu'un placement en devises (différentiel de 2,3 \% en moyenne au deuxième semestre 1991 pour les taux à trois mois). Légèrement supérieur à l'inflation lors de leur lancement (différentiel de 0,6\% au premier trimestre 1991), ce taux est cependant passé en dessous dès le troisième trimestre à cause de son accélération : au quatrième trimestre, l'inflation s'est élevée à $25,4 \%$ alors que le taux d'adjudication des bons du Trésor s'établissait à 19,5\%. 
L'objectif pour l'année 1992-93 est de porter les taux à six mois de $2 \%$ à $4 \%$ au-dessus de l'inflation, tout en maintenant un différentiel avec les placements en devises exprimant la dépréciation attendue de la livre et le risque Égypte.

Ces mesures devraient atténuer les effets inflationnistes des hausses de prix prévues par la politique tarifaire du programme d'ajustement structurel et permettre de ramener l'inflation du pic attendu de $30 \%$ environ en 1991-92 à $9 \%$ en 1993-94, puis à la stabiliser à environ $5 \%$. Elles devraient être complétées à l'avenir par une diversification des instruments monétaires utilisés.

\section{Marché des changes}

o La réforme du marché des changes prévue par le programme de stabilisation a été opérée en deux étapes :

1 - Un double marché des changes a tout d'abord été instauré le 26 février 1991 :

1. Le marché primaire, sur lequel les taux de change étaient fixés quotidiennement par un comité de banquiers, était alimenté en devises par les recettes d'exportations de biens, celles du canal de Suez et de l'oléoduc Sumed ainsi que les prêts et dons publics. Les ressources du marché primaire étaient utilisées pour les dépenses extérieures publiques, y compris le service de la dette.

2. Le marché secondaire sur lequel le taux de change était déterminé par le libre jeu de l'offre et de la demande, la livre étant donc une monnaie flottante sans référence fixe. Suite à l'instauration de ce taux, la monnaie égyptienne a perdu environ $9 \%$ de sa valeur par rapport au dollar, qui s'appréciait simultanément de plus de $10 \%$ contre les monnaies de la $\mathrm{CEE}$, ce qui annulait pratiquement cette baisse.

Aménagement du système précédent, ce système instaurait une période de transition ne devant pas dépasser un an, pendant laquelle la Banque Centrale intervenait pour que la différence entre les deux taux ne dépasse pas $5 \%$.

- L'unification du marché des changes est intervenue le 8 octobre 1991. Cette avance de près de quatre mois sur la date limite fixée a été permise par la stabilité depuis mars 1991, sur le marché libre, du cours de la livre par rapport au dollar, monnaie de référence implicite. Due essentiellement à l'abondance de devises, cette stabilité s'est poursuivie après cette unification, dont la réussite a également été attestée par la disparition complète du marché parallèle.

4 La première agence de change privée indépendante du système bancaire a enfin été inaugurée le 4 août 1991 par le ministre de l'Économie. Selon la nouvelle loi régissant ce type de sociétés, leur capital doit s'élever au minimum à 1 million LE et leurs activités sont limitées aux opérations de change au comptant. Associée à la libération des taux d'intérêt, la libéralisation du marché des changes a en outre eu pour effet de renverser le processus de dollarisation de l'économie.

\section{Réforme bancaire}

35 Public à $90 \%$, le système bancaire égyptien est inadapté à la libéralisation de l'économie. Le programme de stabilisation prévoit l'adoption d'une nouvelle loi bancaire, en cours de discussion au Parlement, dont certaines des dispositions ont 
commencé à être mises en oeuvre, bien que la réforme du système financier soit prévue dans le cadre de la deuxième phase de l'ajustement structurel.

Les ratios de réserves et de liquidités ont d'ores et déjà été fixés à $15 \%$ et $20 \%$ respectivement pour les dépôts en livres et à $15 \%$ et $25 \%$ pour les dépôts en devises. Des mesures visant à lutter contre la sous-capitalisation des banques ont également commencé à être adoptées. Le budget 1990-91 a ainsi pris en charge la recapitalisation des quatre principales banques publiques, pour un montant qui a dépassé 6 milliards LE. Le manque de données concernant le provisionnement des créances douteuses sur les entreprises publiques continuant cependant à jeter un doute quant à la solvabilité d'autres banques, la Banque Centrale a édicté de nouvelles règles sur la classification des créances et sur la capitalisation, basées sur celles de la Banque des Règlements Internationaux. Il est trop tôt pour juger de leurs résultats.

Suite aux problèmes rencontrés par la Bank of Credit and Commerce (Misr), la Banque Centrale a enfin élaboré un mécanisme de garantie des dépôts qui fait jouer la solidarité interbancaire, créant le 27 août 1991 un Fonds de garantie des dépôts financé par des versements des banques commerciales ${ }^{5}$.

Les autres mesures envisagées dans le domaine bancaire visent à le rendre plus solide et plus concurrentiel :

1. Autoriser les filiales de banques étrangères à travailler en livres égyptiennes.

2. Autoriser l'ouverture de nouvelles banques dotées d'un capital correspondant aux standards internationaux (environ 10 millions \$).

3. Faciliter l'ouverture de succursales, dont le nombre est insuffisant (environ 1500 ).

4. Rehausser la productivité en formant et réduisant le personnel des banques publiques et en modernisant leur équipement.

5. Introduire de nouveaux produits bancaires.

6. Renforcer la réglementation et la supervision du système bancaire par la Banque Centrale, qui doit mettre fin à la discrimination entre banques publiques et privées.

7. Privatiser les banques égypto-étrangères et éventuellement certaines banques publiques (la loi 203 ne concerne pas le secteur financier).

\section{Le programme d'ajustement structurel}

Le programme d'ajustement structurel de l'économie est élaboré en collaboration avec la Banque Mondiale, qui a comme à son habitude assorti son premier prêt à l'ajustement structurel (PAS) d'une série de «conditionnalités » devant être remplies pour que sa deuxième tranche soit déboursée. La Banque Africaine de Développement et la CEE utilisent les mêmes critères pour le versement de leurs contributions complémentaires.

Approuvé par le conseil d'administration de la Banque le 19 juin 1991, ce prêt devait commencer à être déboursé en septembre suivant. Il n'a cependant été signé que le 22 novembre, en raison du retard mis par les autorités égyptiennes à remplir les conditions de signature dans les domaines de la réforme du secteur public et des prix du coton.

41 Le retard dans la mise en oeuvre des réformes peut relever de facteurs institutionnels ou même techniques, principalement dus à la faible capacité opérationnelle de l'administration égyptienne. Le ministère de la Coopération internationale par exemple 
est chargé de la mobilisation et de la gestion des ressources extérieures, dont celles liées au programme d'ajustement. Or, il manque de personnel qualifié, notamment au niveau des experts et chercheurs (déficit de $25 \%$ à la fin de 1991). Dans un ministère dont la principale fonction exige une communication constante avec les pays étrangers, seuls quelques cadres supérieurs maîtrisent une langue étrangère. Il partage avec les autres administrations égyptiennes d'autres problèmes de formation et de structure.

C'est pourquoi le Fonds Africain de Développement, filiale de la Banque Africaine de Développement, et le Programme des Nations Unies pour le Développement apporteront de 1992 à 1995 un appui financier et technique au « secrétariat technique pour la mise en oeuvre du programme d'ajustement structurel » créé à la fin de 1991 au sein de ce ministère.

La Banque Mondiale prévoyait le déblocage éventuel de la deuxième tranche de son PAS en juillet 1992, soit au début de l'année financière égyptienne 1992-93. Compte tenu d'un financement rétroactif au 28 février 1991, ce prêt devait être totalement versé à la fin de 1992, soit sur une période de 22 mois coïncidant avec celle du programme de stabilisation du FMI. Suite au retard d'environ trois mois pris par la signature du prêt, le versement de la deuxième tranche pourrait être repoussé. On note que l'échéance du PAS de la Banque Africaine de Développement venant en co-financement est fixée au 30 juin 1993.

Le premier tirage de l'Égypte sur le PAS de la Banque Mondiale n'est intervenu qu'en mars 1992 pour des raisons non divulguées. Ce prêt étant destiné à financer des importations, les autorités égyptiennes ont probablement préféré recourir à d'autres crédits moins onéreux disponibles à cet effet ou même les régler au comptant grâce aux importantes réserves en devises engrangées depuis la guerre du Golfe. Les conditions de prêt de la Banque Mondiale sont en effet onéreuses pour un pays comme l'Égypte qui bénéficie de nombreux crédits à taux préférentiels. Son prêt à l'ajustement structurel est en effet accordé sur 20 ans, dont 5 de grâce, à un taux variable lié aux conditions de financement de la Banque (il était au début de 1992 de 7,7\%, plus une commission annuelle de 0,25\% sur les sommes non déboursées).

45 En Égypte comme ailleurs, l'ajustement structurel vise, au prix de réformes réceptionnistes à court terme, à rétablir des conditions de croissance structurelle à moyen et long termes. Les principales composantes du programme égyptien sont la réforme des entreprises publiques et la privatisation de nombre d'entre elles, la promotion du secteur privé, la libération des prix et la libéralisation du commerce extérieur. La deuxième phase de l'ajustement structurel concernera essentiellement le secteur financier.

\section{Réforme des entreprises publiques}

Composante de base du programme d'ajustement structurel de l'économie, selon les autorités égyptiennes, la réforme des entreprises publiques a été annoncée par le président Moubarak dans son discours du 1er mai 1990. Son coût pour le budget 1991-92 est évalué à 700-800 millions LE, somme dont il faudra retrancher les recettes des privatisations. Elle a pour objectifs de préparer la privatisation à moyen terme des industries non-stratégiques et d'aménager l'environnement légal et institutionnel des entreprises afin de leur permettre de viser la rentabilité. 


\section{Nouveau cadre légal}

Adoptée par le Parlement en juin 1991 et promulguée le 20 juillet suivant, la nouvelle loi 203 régissant le secteur public économique rapproche ses conditions d'activité de celles du secteur privé en accordant une autonomie de gestion aux entreprises publiques et en dissociant la propriété étatique de ces entreprises de leur gestion, grâce à la transformation des organismes publics les coiffant auparavant en holdings sectoriels ${ }^{6}$. Les textes d'application de cette loi ont été publiés avec retard (le 15 octobre), ce que la Banque Mondiale a reproché au gouvernement.

Deux tiers des entreprises publiques doivent avoir été réorganisées selon les termes de cette loi d'ici le 30 juin 1992 et l'ensemble avant la fin de l'année, ce qui permettra de les restructurer et éventuellement de les liquider. Ces holdings publics dépendent d'une nouvelle structure placée auprès de la Présidence du Conseil, le Bureau des Entreprises Publiques, dirigé par une personnalité appelée à avoir le rang de ministre. Ce Bureau n'est pas soumis aux règles habituelles delà fonction publique, ce qui lui permet d'engager des experts privés.

49 Le gouvernement entend par ailleurs présenter en décembre 1992 une nouvelle loi unifiant les conditions de l'activité économique, publique et privée, qui se substituera aux lois 230(1989) et 203(1991). Cette dernière n'est donc qu'une loi transitoire adoptée pour ménager dans un premier temps les partisans d'un secteur public dominant, en attendant la promulgation avant la fin de 1993 de la loi définitive, qui a commencé à être préparée dès 1991.

La nouvelle loi sur les entreprises publiques devait permettre de les privatiser. Si l'on ne joue pas sur les mots, c'est-à-dire si l'on considère qu'une participation privée au capital limitée à $49 \%$ au maximum ne constitue pas une privatisation, cette dernière est interdite par la loi 203. Son rédacteur a utilisé un artifice habile pour l'autoriser légalement malgré cela: si la loi 203 précise que toutes les entreprises publiques doivent passer sous son régime, elle ne leur interdit pas d'en sortir ultérieurement. Leur privatisation sort donc du cadre de cette loi, mais n'est pas interdite, ce qui a pu échapper aux députés l'ayant votés et explique la facilité avec laquelle un Parlement réputé dirigiste l'a adoptée ${ }^{7}$.

\section{Aménagement de l'environnement des entreprises}

51 L'adaptation du cadre législatif est une condition nécessaire, mais non suffisante, à la réussite du passage des entreprises publiques à un fonctionnement d'économie de marché. Outre un changement de mentalité nécessitant des années d'effort, ce dernier dépend également d'un aménagement de l'environnement dans lequel évoluent les entreprises publiques, principalement en matière financière et de main-d'oeuvre.

\section{Réforme financière}

52 L'autonomie des sociétés d'État a tout d'abord pour corollaire la fin du financement budgétaire de leurs investissements, la restriction des crédits accordés par la National Investment Bank (publique) et leur alignement sur les conditions offertes par les autres institutions de crédit, ainsi que la plus grande difficulté d'obtenir la garantie étatique à leurs emprunts et des taux préférentiels auprès des banques commerciales publiques. À partir de juillet 1992, les entreprises publiques auront toute liberté dans le choix de

Égypte/Monde arabe, 9 | 1992 
leur banque, mais devront parallèlement rechercher d'autres modes de financement, tels que rémission d'actions, le réinvestissement de dividendes et les placements financiers. Les conditions de leur accès au crédit seront par ailleurs rendues plus sévères par la Banque Centrale.

Le soutien de l'État et des holdings aux entreprises déficitaires deviendra enfin exceptionnel, limité à des opérations de restructuration. Le Bureau des Entreprises Publiques, en coopération avec les banques créditrices et des consultants extérieurs, évaluera les entreprises en difficulté avant leur passage à l'autonomie afin de les restructurer ou de les liquider.

Les dettes à long terme de certaines entreprises publiques seront converties en participation au capital par l'État, qui prendra en charge leur service. Les pertes cumulées des 17 sociétés placées sous la tutelle du ministère de l'Industrie (sur 116) que ce dernier a estimé déficitaires en 1989-90 s'élèvent à 2 milliards LE environ, dont 200-400 millions LE d'intérêts à prendre en charge par le budget.

\section{Main-d'œuvre}

55 La mobilité de la main-d'oeuvre rendue nécessaire par l'impératif de rentabilité sera soutenue par l'action du Fonds social d'aide à l'ajustement structurel, qui financera les mises à la retraite anticipées, les indemnités de licenciement et le reclassement des licenciés. La loi 203 ne mentionne pas explicitement quels sont les moyens adoptés pour comprimer les effectifs, qui seront précisés dans le cadre de l'activité du Fonds social ou au cas par cas. On note que depuis le début de la décennie 1980, la politique de garantie d'emploi public à tous les diplômés a été abandonnée de facto, sans amendement législatif. Ceci a permis de stabiliser à environ 3,1 millions depuis 1985 le nombre d'employés des entreprises publiques, avec même un déclin dans l'industrie.

On estime que durant les deux exercices budgétaires 1991-92 et 1992-93 $10 \%$ des 1,2 million d'employés des entreprises publiques seront touchés par les compressions d'effectifs (40 000 par semestre à partir du début de 1992). Au premier semestre 1992, $50 \%$ recevront une allocation de chômage équivalente à $80 \%$ de leur salaire et $20 \%$ seront mis à la retraite anticipée et toucheront $60 \%$ de leur salaire. Compte tenu d'un salaire mensuel moyen évalué à $165 \mathrm{LE}$ et d'une indemnité de licenciement représentant six mois de salaire, les compressions d'effectifs dans les entreprises publiques représenteront une charge de 60,2 millions LE pour le budget 1991-92, à comparer aux 39,6 millions LE de salaires économisés, soit une dépense nette pour l'État de 20,6 millions LE.

57 Le coût de la formation des employés des entreprises publiques restructurées est évalué quant à lui à 120 millions LE. La loi 203 abroge par ailleurs la loi 48 sur l'égalité des rémunérations des entreprises publiques, qui deviennent libres de fixer les salaires de leurs employés. La libération des prix et la libéralisation du commerce extérieur (cf. infra) achèveront de mettre sur le même plan les entreprises publiques et privées.

\section{Privatisations}

58 L'Égypte a commencé à privatiser certaines activités dès 1986, puis a accéléré cette politique après le discours présidentiel du 1er mai 1990. Dans le cadre du programme d'ajustement structurel, le gouvernement a arrêté un plan de privatisations pour les 
deux années fiscales 1990-91 et 1991-92, qui comporte cependant peu d'échéances et d'engagements quantitatifs précis. Il se répartit sectoriellement comme suit ${ }^{8}$ :

\section{- Industrie, BTP :}

1. Vente ou location des actifs oisifs ou marginaux des entreprises entièrement publiques : au début de février 1992, un total de 457 millions LE d'actifs appartenant à 34 sociétés publiques étaient cédés ou en cours de cession et 10 sociétés publiques avaient loué des facteurs de production.

2. Cession partielle ou totale de la part étatique dans les entreprises conjointes (joint-ventures) formées avec des partenaires privés, notamment étrangers, dans le cadre des lois 43 (1974) et 230 (1989) : aux quelque 2,6 milliards LE de participations publiques dans les 245 jointventures recensées au premier semestre 1991 viendront s'ajouter les parts de l'État égyptien dans la centaine d'entreprises conjointes alors en cours de formation. L'officieux Al-Ahrâm a publié une liste de 192 joint-ventures privatisables dans ses éditions des 31 janvier et 7 février 1992.

3. Augmentation de la part du secteur privé au capital des 393 sociétés entièrement publiques, au capital évalué au premier semestre 1991 à 124 milliards LE, par vente d'actions ou par augmentation de capital: toute société régie par la loi 203 ne peut avoir de participation privée supérieure à $49 \%$ de son capital, auquel cas elle sort du cadre de cette loi. Le Bureau des Entreprises Publiques, organisme placé auprès du Premier ministre, et les holdings, qu'il supervise, détermineront en fonction des possibilités du marché quelles parts de chacune de ces entreprises seront proposées aux investisseurs. Si ces derniers répondent favorablement 
aux sollicitations de l'État, toutes ces sociétés seront privatisables, à l'exception des secteurs de souveraineté (hydrocarbures, canal de Suez, armement).

Le gouvernement, qui s'était engagé à publier une première liste d'entreprises privatisables avant la fin de 1991, ne l'a fait que le 26 janvier 1992, au moment de la visite au Caire d'une mission de la Banque Mondiale qui a critiqué publiquement la lenteur du processus. Les autorités avaient dès l'origine identifié les obstacles suivants à sa mise en oeuvre rapide :

1. La difficulté d'estimer la valeur des entreprises et de procéder à leur assainissement financier préalable à la vente.

2. Le grand nombre d'entreprises à privatiser par rapport à la demande potentielle.

3. L'inadaptation du marché financier.

4. Le manque de transparence des transactions.

5. Les problèmes posés par la main-d'oeuvre excédentaire et les actifs non rentables.

6. La complexité de promouvoir un actionnariat populaire afin d'éviter la concentration des richesses.

7. La difficulté d'établir le climat de confiance auprès des investisseurs égyptiens et étrangers envers la politique de libéralisation.

8. La faiblesse des capacités locales d'encadrement du processus.

9. Le programme du gouvernement cite enfin des «contraintes politiques» susceptibles d'entraver le processus, sans autre précision. La Banque Mondiale estime quant à elle qu'« il pourrait avorter en raison d'oppositions au sein du gouvernement et de la fonction publique ».

Le programme de privatisations et de liquidation d'entreprises publiques de l'année budgétaire 1992-93 devait être arrêté avant avril 1992. Si tel a été le cas, il n'a pas encore été rendu public. Les instruments d'incitation aux investisseurs doivent par ailleurs être définis d'ici juin 1992 afin d'être opérationnels en septembre 1992. Une mission de la Banque Mondiale devait effectuer une première évaluation du programme avant la fin mars 1992.

D'autre part, l'unification en 1993 des règles de l'activité économique permettra d'ôter tout obstacle légal à la privatisation majoritaire des entreprises soumises à la loi 203 et aux holdings de privatiser une partie de leur capital, ce qu'elle interdit pour le moment.

\section{Promotion du secteur privé}

Le programme d'ajustement vise à mettre fin à la discrimination entre secteurs public et privé, afin qu'augmente la part de ce dernier au PIB.

La procédure d'agrément gouvernemental aux investissements a permis de protéger les entreprises existantes, publiques ou privées, contre la concurrence. De nombreuses mesures préférentielles ont par ailleurs favorisé le secteur public. L'agrément est devenu automatique en juillet 1991 pour tous les investissements, y compris les extensions d'unités, sauf ceux placés sur " une liste négative » (voir encadré) qui sera réduite en juillet 1992, puis abolie. Cette procédure a été remplacée par une simple déclaration à l'administration, qui doit enregistrer le projet dans les 15 jours et accorder la licence de production dans les 30 jours suivant cette déclaration. Les textes 
d'application des lois 43 et 230 régissant les investissements étrangers ont été amendés à cet effet.

La « liste négative » des investissements privés commencé à être aboli en mai 1991 avec la liquidation de l'Organisme public de commercialisation du ciment. En juillet 1991, les usines ont été autorisées à vendre $20 \%$ de leur production à des distributeurs privés, pourcentage devant être doublé en un an, puis porté à 100 \% d'ici 1995. personnel par l'intermédiaire des bureaux de main-d'oeuvre publics et les agences privées de recrutement sont désormais autorisées.

\section{Politique tarifaire}

78 La levée progressive du contrôle des prix permettra d'alléger les subventions de l'État à l'économie et de libéraliser cette dernière. Le programme d'ajustement vise à accélérer les mesures prises ces dernières années en la matière.

\section{Agriculture}

L'agriculture est le secteur de l'économie où la libéralisation est la plus avancée. Le ministère de l'Agriculture et de la Réforme agraire a éliminé à partir du milieu de la décennie 1980 les contrôles sur les cultures, les prix et la commercialisation des denrées agricoles, et a lancé un programme de privatisation des domaines étatiques et des activités para-agricoles.

Pour compléter les mesures déjà entreprises, le gouvernement s'est engagé dans le cadre du programme d'ajustement structurel à réduire de moitié les subventions aux aliments pour animaux, aux engrais et aux pesticides à l'issue de l'année financière 1991-92, puis à les supprimer durant l'année suivante à l'exception des pesticides destinés au coton, pour lesquels un programme quadriennal de suppression progressive 
doit être lancé en 1991-92. La commercialisation de ces produits est appelée à être confiée au secteur privé.

81 Le coton bénéficiera de conditions particulières en raison de son importance pour l'industrie et les exportations de l'Égypte. L'objectif est de supprimer tout contrôle étatique qui ne soit pas strictement technique à l'horizon 1995-96, l'incitation par les prix de vente devant suffire à assurer les quantités adéquates. Durant la période d'ajustement, le gouvernement fixera pour chacune des sept principales qualités de coton produites des prix différenciés avec pour objectif d'atteindre des prix au producteurs basés sur les prix internationaux. Les prix ont été relevés à cet effet en mars 1991, s'étageant de 40 à 100 LE/quintar selon les qualités pour la saison 1991-92. Ceci représente $60 \%$ des prix internationaux, chiffre devant être porté à $67 \%$ en juillet 1992, contre $55 \%$ en 1990-91. Les prix fixés en début de saison seront ajustés en fonction de l'évolution du taux de change de la livre. Le coton vendu aux filatures le sera à un prix couvrant au minimum les coûts de production, d'égrenage et de transport. Les restrictions à l'importation seront levées afin de faire jouer la concurrence.

\section{Énergie}

82 Les prix moyens pondérés des produits pétroliers sur le marché égyptien doivent être portés d'ici 1995 à l'équivalent des prix internationaux, soit la moyenne annuelle des prix fob à la sortie des raffineries italiennes, selon le calendrier suivant : mai 1991, $45 \%$ des prix internationaux ; décembre 1991, $56 \%$; mai 1992, $67 \%$; mai 1993, $78 \%$; mai 1994, $89 \%$; juin 1995, $100 \%$. La première hausse intervenue en mai 1991 a augmenté les prix égyptiens de $53 \%$, mais la deuxième augmentation opérée en janvier 1992 n'a touché que certains produits, en dépit de ces engagements ${ }^{10}$.

83 D'ici juin 1995 également, les prix du gaz naturel vendu aux industriels devront être alignés sur ceux du fuel sur le marché intérieur et les prix de l'électricité couvrir les coûts de production marginaux à long terme. Une première augmentation de $50 \%$ des prix de l'électricité est intervenue en mai 1991, ce qui les a portés à $59 \%$ de l'objectif tarifaire assigné. Quatre autres augmentations annuelles de $10 \%$ doivent suivre.

Ces hausses sont destinées à ramener le total des subventions implicites de l'État à l'énergie de 9,6 \% du PIB en 1990-91 à 6,1 \% en 1991-92.

\section{Secteur public industriel}

85 Les prix des produits industriels fabriqués par le secteur public seront tous libérés d'ici juillet 1992 à l'exception de certains produits alimentaires de base, des cigarettes (monopole étatique), des filés de coton et de certains produits non compétitifs, dans un premier temps. La protection de ces derniers contre la concurrence étrangère sera supprimée en 1994, date prévue de l'abolition des barrières quantitatives à l'importation, et leur subvention indirecte par les inputs, énergétiques notamment, cessera d'ici juin 1995.

Les subventions à la commercialisation du ciment et des engrais seront supprimées progressivement d'ici juin 1995. Durant la période transitoire au cours de laquelle coexisteront les deux modes de distribution, le prix de ces produits vendus par le secteur public sera calculé sur la base de leur équivalent importé, plus les droits de douane. 


\section{Transports}

L'Egyptian National Railways augmentera ses tarifs au début de chaque année afin d'améliorer de $5 \%$ par an à partir de 1990-91 son ratio revenus propres/dépenses, de telle sorte qu'il atteigne $100 \%$ en 1997-98. Une première augmentation de $15 \%$ à $40 \%$ des tarifs voyageurs et de $15 \%$ des tarifs marchandises est intervenue en janvier 1991 . Le gouvernement s'est engagé à ce que le capital de la société soit consolidé à hauteur de l'encours de sa dette à long, terme au 30 juin 1990, soit 2857 millions LE, à prendre en charge le service de cette dette et à couvrir ses investissements jusqu'en 1997-98 par des dotations annuelles en capital.

Les compagnies parapubliques de transport routier de voyageurs et de marchandises acquerront dans un premier temps une autonomie de gestion leur permettant de fixer elles-mêmes leurs tarifs et besoins en personnel, avant d'être privatisables.

\section{Libéralisation du commerce extérieur}

9 La politique poursuivie vise à éliminer les obstacles non tarifaires aux importations et les distorsions en matière de droits de douane, afin de renverser les tendances protectionnistes des trente dernières années et de soumettre en conséquence l'économie égyptienne à la concurrence internationale.

En juin 1991, 25,6\% de la production intérieure égyptienne était protégée par des obstacles non tarifaires aux importations et $4,2 \%$ était touchée par des restrictions de ce type à l'exportation, contre respectivement 52,5 \% et 11,9 \% en mars 1990 .

Engagée en mars 1990, la levée des obstacles aux importations sera accélérée. De cette date à la mi-1991, la valeur de la production agricole et industrielle protégée par des interdictions d'importation a été réduite de 3.554 millions LE, soit de $37,2 \%$ du total à $22,7 \%$. D'ici au déblocage de la seconde tranche du PAS, le nombre de produits interdits à l'importation sera abaissé afin de ne pas dépasser $30 \%$ des produits agricoles et industriels protégés de la sorte en mars 1990, soit 10,6 \% de leur valeur globale à cette date. Ceci équivaut à une réduction supplémentaire de 2.730 millions LE de la valeur de la production protégée. Certains secteurs hautement protégés, notamment les industries du textile et du cuir, ne seront toutefois touchés qu'ultérieurement.

2 Des mesures anti-dumping (hausses de certains droits de douane) seront prises simultanément au démantèlement des barrières non tarifaires, afin de donner le temps aux entreprises de s'adapter à la concurrence internationale.

Les autres réformes que le gouvernement s'est engagé à adopter avant le déboursement de la seconde tranche du PAS en matière de commerce extérieur sont les suivantes :

1. Lever 4 des 18 agréments préalables à l'importation existant au début du programme (contre 55 en mars 1990).

2. Resserrer les marges de variation des droits à l'importation entre un plancher de $10 \%$ et un plafond de $80 \%$, excepté pour certains produits alimentaires de base et les articles de luxe, contre des amplitudes de $5 \%$ à $100 \%$ à la veille du programme et de $0,7 \%$ à $120 \%$ avant mars 1990.

3. Supprimer les règles de contrôle de la qualité à l'importation non liées au respect des normes. 
4. Présenter devant le Parlement une loi éliminant 27 des 90 articles qui jouissaient en mars 1990 de tarifs préférentiels à l'importation (27 l'avaient déjà été entre cette date et le début du programme).

5. Opérer des augmentations annuelles de $15 \%$ des quotas à l'exportation des quatre derniers produits soumis à des restrictions de ce type (laine brute, déchets de laine, de coton et de papier) et autoriser l'exportation des six derniers produits interdits. En mars 1990, 20 produits étaient interdits à l'exportation et 17 touchés par des quotas.

6. Améliorer les conditions matérielles et administratives d'admission temporaire et de rétrocession des droits de douane pour les marchandises destinées à la réexportation (inputs notamment).

7. Éliminer les allocations gouvernementales de devises aux entreprises publiques, qui jouiront des mêmes conditions que les sociétés privées pour leurs importations.

94 À moyen terme, toutes les autorisations préalables à l'importation seront levées (sauf cas de sécurité nationale ou de protection sanitaire, écologique, de la moralité publique ou du patrimoine national), les restrictions aux importations et aux exportations continueront à être éliminées et les activités des sociétés privées de commerce international seront favorisées.

\section{Protection de l'environnement}

Une des spécificités du programme égyptien d'ajustement structurel est d'inclure la lutte contre les principaux problèmes du pays en matière d'environnement :

1. Rareté et pollution des ressources en eau.

2. Dégradation et appauvrissement des terres agricoles.

3. Pollution de l'air dans les villes.

4. Réduction des ressources énergétiques.

5. Érosion du littoral.

6. Dégradation du patrimoine naturel et culturel.

7. Gestion des déchets solides et dangereux.

Conditionnalités du prêt d'ajustement structurel 
104 - Application de la loi 203 aux deux/tiers des entreprises publiques. Plan de restructuration ou de liquidation partielle ou totale des entreprises publiques par leurs holdings-mères et le Bureau des Entreprises Publiques.

105 - Mise en œuvre du programme 1991-92 de privatisations et définition du programme 1992-93.

106 - Promulgation d'un décret autorisant les sociétés publiques produisant des engrais et du ciment à céder jusqu'à $40 \%$ de leur production à des distributeurs privés dès début juillet 1992.

107 - Respect du calendrier d'augmentation des prix des produits énergétiques et des tarifs ferroviaires.

108 - Augmentation des prix du coton payé au producteur à hauteur de $66 \%$ des prix mondiaux pour la saison 1992, suppression de la moitié des subventions aux engrais et pesticides durant l'année budgétaire 1991-92 et engagement à les faire disparaître l'année suivante.

109 - Réduction de la protection de la production agricole et industrielle par des interdictions d'importations à hauteur de $10,6 \%$ du total commercialisé, soit une baisse de $70 \%$ de la protection par rapport à la période de base (mars 1990).

110 - Libération des prix des produits industriels du groupe IV et réduction de leur protection douanière ; révision des prix des produits du groupe $\mathrm{V}$ sur la base des coûts de revient et essai de réduction de la quantité de produits au prix centralement contrôlé.

111 - Adoption des mesures relatives à la libéralisation du commerce extérieur.

112 - Accélération du programme de mobilité de la main d'oeuvre financé par le Fonds social.

\section{Le fonds social de développement}

113 Le Fonds social d'aide à l'ajustement structurel de l'économie égyptienne («Fonds Social de Développement ») vise à faciliter sa réussite. Sans être intégré au programmé d'ajustement, il en constitue un complément indissociable, selon ses concepteurs à la Banque Mondiale, qui reconnaissent ainsi de façon implicite les insuffisances des programmes traditionnels.

114 Si l'on retient l'hypothèse selon laquelle le Fonds social n'est qu'un élément politique destiné à emporter la signature par le gouvernement égyptien des programmes du FMI et de la Banque Mondiale, on aboutit à la même conclusion. La réussite de l'ajustement dépend en effet en premier lieu des capacités politiques de l'État à le mettre en oeuvre. Les crédits du Fonds social distribués par l'État pour apaiser le mécontentement populaire provoqué par l'ajustement renforcent l'État, ce qui accroît son efficacité dans sa politique de réformes.

Un des paradoxes de la libéralisation est en effet qu'elle dépend d'une forte impulsion de l'État, intermédiaire entre la société et les institutions financières internationales. Le coût d'un tel fonds peut se révéler inférieur à celui induit par des difficultés d'ordre social susceptibles d'entraver la mise en oeuvre de l'ajustement ou même à la facture d'éventuelles émeutes. Les autorités égyptiennes, qui ont une expérience incomparable de l'aide internationale, n'ont probablement pas manqué de faire valoir cela afin 
d'assurer le couplage du programme de réformes économiques avec la constitution d'un Fonds social doté de ressources conséquentes.

Instruite par les problèmes sociaux posés par l'ajustement dans certains pays, la Banque Mondiale avait déjà expérimenté avec succès en Bolivie cet instrument d'accompagnement. Le fonds prévu pour l'Égypte est de taille nettement supérieure, ce qui constitue une nouvelle indication du traitement de faveur réservé par la communauté internationale à ce pays, par rapport aux autres ayant opté pour l'ajustement.

\section{L'état des financements}

La Banque Mondiale ayant commencé à préparer ce projet en octobre 1989, ce traitement de faveur était envisagé avant la crise du Golfe, mais cette dernière lui a conféré toute son ampleur. Le projet retenu en avril 1990 portait sur un fonds de 200 millions $\$$, alors que la dotation minimale prévue dans le projet proposé aux bailleurs de fonds le 17 février 1991 s'élevait à 400 millions \$ et que le rapport présenté le 19 juin 1991 devant le conseil d'administration de la Banque Mondiale pour adoption du projet prévoyait des contributions totales de 572 millions $\$$.

118 L'Association Internationale de Développement (AID), filiale de la Banque Mondiale, chargée du projet, a approuvé le 19 juin 1991 et signé le 27 septembre suivant un prêt de 105 millions de DTS (140 millions \$), sur 35 ans dont 10 de grâce, sans intérêt mais avec une commission annuelle de $0,75 \%$ sur les fonds versés et de $0,5 \%$ sur le restant, ce qui ouvrait la voie aux autres contributeurs. Bien que l'Égypte soit classée dans la catégorie des pays «à revenu intermédiaire » avec un PIB par habitant estimé à 675 \$ en 1989, elle était ainsi pour la première fois depuis 1981 éligible aux crédits de l'AID, réservés aux pays les plus pauvres. Cette nouvelle éligibilité était motivée par le fait que son PIB par habitant avait chuté de 750 \$ en 1985-86 à 640 \$ en 1989-90 et devait continuer à baisser pendant environ trois ans. Selon le gouvernement égyptien, les engagements de prêts et dons au Fonds social atteignaient 609,45 millions \$à la fin janvier 1992, soit plus du triple des sommes initialement prévues, répartis comme indiqué sur le tableau 2 (voir p. suivante).

119 La Banque Mondiale estimait au moment de l'adoption du projet à environ 3,4 milliards \$ le coût pour l'Égypte de la crise du Golfe en 1990 et 1991, ce qui explique cette augmentation et le fait que le Fonds social n'ait plus pour seuls objectifs de «minimiser les effets des réformes sur les pauvres» et de «faciliter la mobilité de la main-d'œuvre ", mais aussi "d'aider à réinsérer les rapatriés des pays du Golfe ». Les versements du Fonds doivent être répartis sur six ans, de l'année financière 1991-92 à 1996-97.

\section{Les projets retenus}

120 Au début de mars 1992, trois projets d'un montant global de 154,3 millions LE avaient reçu une approbation définitive du Conseil d'administration et trois autres une approbation de principe. Les dépenses totales prévues s'élevaient alors à 219,28 millions LE, soit 66,45 millions $\$$ ou $10,9 \%$ des contributions étrangères prévues (voir tableau 3 page suivante).

Ces projets s'inscrivent dans le cadre des programmes définis par l'AID pour le Fonds social : 
122 - Infrastructure publique. 255 millions \$ alloués au soutien des services publics locaux en période de restrictions budgétaires, grâce à des travaux d'intérêt public qui résorberont par ailleurs le chômage. Ce programme devrait créer 26000 emplois temporaires dans le secteur du BTP et 15.000 emplois permanents dans les travaux d'entretien.

123 - Soutien aux compagnies de transport urbain. Allocation de 53 millions \$ à l'achat de pièces détachées et de véhicules par les deux sociétés publiques d'autobus du Caire et d'Alexandrie.

Tableau 2 : Contributions au Fonds Social de Développement (millions \$)

\begin{tabular}{|c|c|c|}
\hline Nature & Montant & Recottes fiscolies \\
\hline Prốts & & \\
\hline AID & 140,00 & 1,98\% dintênát en LE. Approuvé par le gouvernement \\
\hline Allemagne & 30,00 & $0,75 \%$. En deutsche marks. Approuvé. \\
\hline $\begin{array}{l}\text { Fonds Koweitien de } \\
\text { Développement }\end{array}$ & & $\begin{array}{l}1.5 \% \text { sur les sommes déboursées, plus } 0.5 \% \text { sur le } \\
\text { principal non débourse. Approuvé et ratifié par le }\end{array}$ \\
\hline Economique Arabe & 50,00 & Parlement \\
\hline $\begin{array}{l}\text { Fonds d'Abou Dhabi } \\
\text { Fonds Arabe de }\end{array}$ & 50,00 & $\begin{array}{l}\text { Mômes conditions. En dirhams des Emirats. Approuvé. } \\
3 \% \text { sur les sommes débourses, plus } 0,5 \% \text { sur les }\end{array}$ \\
\hline $\begin{array}{l}\text { Développement } \\
\text { Economique et Social }\end{array}$ & 50,00 & $\begin{array}{l}\text { sommes non déboursées. En dinars koweitiens. } \\
\text { Approuvé et ratifíb. }\end{array}$ \\
\hline Sous-total 1 & 320,00 & \\
\hline Dons & & \\
\hline Autriche & 5,00 & Approuve ot ratifié. \\
\hline Danemark & 1,50 & Approuvé. En couronnes. Aiput éventuel de $8,5 \mathrm{M} . \mathrm{s}$ \\
\hline CEE & 153,00 & $\begin{array}{l}\text { Approuvé et ratifié. En LE. } 512 \text { millions LE versés sur } \\
\text { un comple spéciel à la National Bank of Egypt. }\end{array}$ \\
\hline Pays-bas & 9,30 & Approuve ot ratifié. En floring \\
\hline Canada & 3,00 & En dollars canadiens. \\
\hline Stuisse & 30,00 & \\
\hline Suède & 10,00 & \\
\hline France & 10,00 & \\
\hline Etat-Unis & 55,00 & $\begin{array}{l}\text { Approuvié et signé. En LE. } 180 \text { millions LE versés à la } \\
\text { Banque Centrale. }\end{array}$ \\
\hline Irlande & 4,00 & Approuvé. \\
\hline Norvège & 2,00 & \\
\hline Programme des Nations-Unies & & \\
\hline pour le Développement & 4,00 & Assistance tochnique. \\
\hline Autres & 2,65 & En LE. 8,8 millions LE versés. \\
\hline Sous-1otal 2 & 289,45 & \\
\hline Total & 609,45 & \\
\hline
\end{tabular}

N. B. Les montants prévus en livres égyptiennes sont des fonds de contrepartie. Source : Fonds Social de Développement 
Tableau 3 : Projets approuvés par le Fonds Social de Développement

\begin{tabular}{|c|c|c|c|}
\hline Projets & Programme & Finarcement & Montan \\
\hline 1. Approbation définitive & & & \\
\hline Aménagement des rives du Nil & InIrastruclure publique & Dons & 71,28 \\
\hline $\begin{array}{l}\text { Aide à rartisanat dans } 4 \\
\text { gouvernorats }\end{array}$ & Développement social & $\begin{array}{l}\text { Dons, administrés } \\
\text { par des ONG }\end{array}$ & 15,00 \\
\hline & & $\begin{array}{l}\text { Dons, administrés } \\
\text { par des ONG }\end{array}$ & 68,00 \\
\hline 2. Approbation de principe & & & \\
\hline Crédịts à l'agriculture & $\begin{array}{l}\text { Aide aux petites } \\
\text { entreprises }\end{array}$ & Prêts & 30,00 \\
\hline Crédils aux petites entreprises & $\begin{array}{l}\text { Aide aux petiles } \\
\text { entreprises }\end{array}$ & Préls & 20,00 \\
\hline Aménagement utbain à Mansoura & Intrastructure publique & Dons & 15,00 \\
\hline Total & & & 219,28 \\
\hline
\end{tabular}

Source : Fonds Social de Développement, février 1992.

124 - Développement social. 42 millions \$ pour l'appui aux soins de santé primaire, à la PMI, aux programmes de vaccination, de nutrition, d'alphabétisation et de formation et aux activités productives auto-organisées.

- Aide aux petites entreprises (employant moins de 15 personnes et dotées d'équipements d'une valeur maximale de 10000 \$). Attribution de 120 millions \$ de prêts au taux du marché, avec assistance technique et formation, pour la création de 70000 petites entreprises par les rapatriés du Golfe et les chômeurs et l'extension des petites entreprises existantes.

126 - Plan transitoire pour l'emploi. Le financement des retraites anticipées, des allocations de chômage et des aides à la réinsertion des travailleurs licenciés dans le cadre du programme d'ajustement structurel, ainsi que de la formation des employés des entreprises publiques restructurées, incombe au Fonds social. Montant prévu: 91 millions \$.

127 - Assistance technique. D'un coût de 11 millions \$, ce projet d'appui à l'action du Fonds social vise à améliorer l'évaluation et la réalisation de projets sociaux ainsi qu'à développer la collecte des données socio-économiques.

\section{Un organisme égyptien ou égypto-étranger?}

128 Le projet de Fonds Social de Développement a été préparé par l'Association Internationale de Développement, puis il a été institué par décret présidentiel du 29 janvier 1991 en tant qu'organisme de droit égyptien, gestionnaire des contributions qu'il reçoit de l'étranger en majorité. La participation du gouvernement égyptien au 
Fonds est partiellement couverte par l'équivalent en livres des 55 millions $\$$ de fonds de contrepartie (de la vente en Égypte de dons en nature) constituant la contribution américaine.

Sa gestion doit suivre des procédures prédéfinies avec la Banque Mondiale et chaque projet doit être supervisé conjointement par l'État égyptien et l'AID. Des budgets trimestriels et annuels doivent être fournis au ministère égyptien des Finances, et ses opérations doivent être contrôlées chaque année par des audits agréés par l'AID.

Le Fonds social est administré par un conseil de huit membres, nommés le 6 mai 1991. Formé à l'origine des ministres des Affaires sociales, des Affaires du Cabinet et du Développement administratif, du Travail et de la Formation et de la Coopération internationale ainsi que de représentants de la Fédération des Industries, de l'Association des hommes d'affaires d'Alexandrie, de la Société des Investisseurs de Dix de Ramadan et de la Fédération du Tourisme et des Hôtels, le conseil d'administration a été élargi par la suite à dix-sept membres. Ces derniers sont désignés par le Premier ministre, qui nomme également son directeur général. M. Hamed Moubarak, cousin du Président, a été nommé à ce poste en février 1991, mais a dû en démissionner le 24 novembre suivant en raison des critiques adressées envers le fonctionnement du Fonds. Un directeur intérimaire, M. Hussein Gamal, était en place à la fin mars 1992. Ce n'est que le 29 mars 1992, un an et deux mois après sa constitution et avec six bons mois de retard sur le calendrier fixé à l'origine, que le Fonds débloquait son premier financement, un prêt de 7 millions LE aux petites entreprises des gouvernorats de Charqiya, du Fayyoum, d'Assiout et d'Isma'iliya.

Plusieurs facteurs sont à l'origine des problèmes du Fonds social, qualifiés «d'administratifs" par le ministre de la Coopération internationale, M. Maurice Makramallah :

1. La concurrence entre les ministres du Plan, de la Coopération internationale et des Affaires du Cabinet pour assurer la tutelle du Fonds social, finalement confiée au Premier ministre, comme pour le Bureau des Entreprises Publiques.

2. L'intention du gouvernement de faire supporter au Fonds social la charge du remboursement des prêts en devises qu'il reçoit, ce que ses bailleurs de fonds considèrent comme allant à rencontre de ses objectifs.

3. La lourdeur de fonctionnement du conseil d'administration, qui devrait selon la Banque Mondiale être épaulé par une structure de suivi de ses décisions. Ce n'est qu'au début de 1992 que le gouvernement a mis en place cinq sous-comités visant une plus grande efficacité.

4. La réticence des autorités à accepter d'associer aux décisions des organisations nongouvernementales (ONG) égyptiennes.

5. Leur refus d'autoriser la création au Caire d'une cellule de supervision des projets, ce qui irait au-delà des contrôles internationaux habituels. L'AID a finalement obtenu en 1992 d'avoir un représentant qu Caire à cet effet.

132 Les principales divergences tiennent donc au fait de savoir si ce fonds est un organisme égyptien, ce qui est juridiquement exact, ou égypto-étranger, ce que son financement implique. Les principes de fonctionnement du Fonds avaient pourtant été arrêtés entre l'AID et l'Égypte avant la signature de l'accord les liant. Cela n'a apparemment pas suffi à chasser l'ambiguïté fondamentale de cet instrument, technique dans son fonctionnement mais politique dans ses objectifs. 
Après des négociations infructueuses, une rencontre entre le gouvernement égyptien et les donneurs d'aide a été convoquée les 9-10 avril 1992 à Bruxelles afin de concilier ces deux facteurs.

\section{Les perspectives macro-économiques de l'Égypte}

Les tableaux 4 à 6 (voir pages ci-après) indiquent l'évolution prévue de l'économie égyptienne en cas d'application du programme de stabilisation et d'ajustement structurel. Commentées ci-dessous, les prévisions qu'ils délivrent sont basées sur des statistiques recueillies au premier semestre 1991, avant la conclusion de l'accord avec le Club de Paris.

Il faut cependant garder à l'esprit que les perspectives de réussite du programme de stabilisation et d'ajustement dépendent de données extra-économiques et de facteurs économiques exogènes, tels que les prix du pétrole, les rapatriements des travailleurs émigrés et les recettes du canal de Suez. Pour réduire leur importance, la politique économique vise à promouvoir les exportations de biens manufacturés par le secteur privé principalement.

136 Cette politique ne pouvant pas donner de résultats immédiats, le produit intérieur brut devrait diminuer en termes réels de $1,5 \%$, puis $3 \%$ pendant les deux premières années de l'ajustement (après une première baisse de $0,5 \%$ en 1989-90) et ne retrouver le chemin de la croissance qu'à partir de 1993-94. Compte tenu de l'accroissement démographique, la consommation réelle par tête devrait chuter de $13 \%$ pendant les premières années de l'ajustement et ne recommencer à augmenter qu'en 1995-96.

Le ratio investissements bruts/PIB devrait être ramené de $23,1 \%$ en 1990-91 à $19 \%$, puis $16 \%$ au cours des deux années suivantes, marquées ainsi par la récession. Il devrait se redresser en 1993-94 (18\%) et tendre vers $25 \%$ à la fin de la décennie.

Le ratio investissements privés/PIB devrait chuter de $7 \%$ en 1990-91 à $4 \%$ l'année suivante, puis remonter à $8 \%$ en 1993-94 pour tendre vers $17,5 \%$ en 1997-98.

La balance commerciale devrait rester structurellement déficitaire. La baisse attendue des recettes d'exportations pétrolières, en dépit d'une légère hausse prévue des prix du pétrole, sera plus que compensée par l'augmentation des autres exportations après la période de récession. Les exportations de produits manufacturés (hors textiles) doivent progresser de $9 \%$ par an en termes réels, celles de textiles de $7 \%$ et celles de coton de $6 \%$. Ces hausses entraîneront une augmentation des importations de matières premières et biens intermédiaires, estimée à $4 \%$ par an après la période de récession, et surtout de produits manufacturés (+ $12 \%$ de 1993 à 1996).

140 Le déficit du compte courant devrait baisser de $10 \%$ en $1991-92$ et chuterait de 3,2 milliards \$ en 1990-91 à 2 milliards \$ en 1993-94, pour remonter ensuite à 3 milliards \$. Un excédent inattendu de 1 milliard \$ est en fait apparu à l'issue de l'année calendaire 1991, mais il est lié à des facteurs exceptionnels (aides et transferts suite à la crise du Golfe, remises de dettes) et devrait donc rester tel. 
Tableau 4 : Projections macroéconomiques (en pourcentages)

\begin{tabular}{|l|r|r|r|r|r|}
\hline & 1989 & 1990 & $1991-93$ & $1994-97$ & $1995-2000$ \\
\hline Croissance et inflation & & & & & \\
Croissance du PIB & 2,4 & 1,3 & $-0,9$ & 3,4 & 3,8 \\
- Agriculture & 2,0 & 1,5 & 2,6 & 3,0 & 3,0 \\
- Pétrole & $-2,8$ & $-2,0$ & $-0,6$ & $-0,1$ & $-0,1$ \\
• Industrie manulacturìre & $-1,4$ & $-2,5$ & $-0,2$ & 5,5 & 5,5 \\
Croissance du PIB & 3,4 & $-1,1$ & $-3,2$ & 1,2 & 1,6 \\
Croissance de la consommation $\mathrm{n}$ & 1,9 & $-1,4$ & $-2,3$ & $-0,7$ & 0,6 \\
Investissements bruts/PIB & 23,6 & 23,1 & 17,7 & 23,1 & 24,4 \\
Epargne intérieure/PIB & 10,2 & 11,0 & 5,9 & 12,2 & 13,7 \\
Inflation & 19,1 & 20,0 & 21,0 & 6,3 & 5,2 \\
\hline Batance des palements & & & & & \\
Croissance des exporlations & 0,0 & 7,7 & 4,2 & 4,2 & 4,3 \\
Croissance des importalions & $-4,1$ & 2,3 & $-0,3$ & 3,9 & 3,6 \\
Solde courant (milliards $\$$ ) & $-2,7$ & $-2,5$ & $-2,7$ & $-2,6$ & $-3,1$ \\
Solde courant /PIB & $-7,2$ & $-6,7$ & $-9,1$ & $-7,9$ & $-8,1$ \\
Intéréts/PIB & 6,6 & 7,6 & 9,1 & 8,2 & 7,7 \\
\hline
\end{tabular}

N.B. 1989 signifie année financière 1988-89 et ainsi de suite.

Source : Banque Mondiale, juin 1991

Tableau 5 : Évolution des principaux indicateurs

\begin{tabular}{|c|c|c|c|c|c|c|c|c|c|c|c|c|}
\hline & 1989 & 1990 & 1991 & 1992 & 1993 & 1994 & 1995 & 1996 & 1997 & 1998 & 1999 & 2000 \\
\hline PIB (milliands \$) & 38,3 & 37,6 & 30,7 & 28,1 & 28,7 & 29,7 & 31,4 & 33,9 & 36,8 & 39,8 & 42,8 & 46,0 \\
\hline Croissance du PIB & 2,4 & 1,3 & $-1,5$ & $\cdot 3,0$ & 1,7 & 2,7 & 3,0 & 4,0 & 4,0 & 4,0 & 4,0 & 4,0 \\
\hline Croissance du PNB & 10, & $.2,0$ & $.4,3$ & $.4,4$ & $.0,3$ & 0,8 & 0,9 & 1,9 & 1,4 & 2,4 & 1,8 & 1,9 \\
\hline Croissance de la consommation ith & 1.9 & $-1,4$ & 2,5 & $-4,8$ & $-4,5$ & $-3,5$ & $-1,4$ & 0,9 & 1,3 & 0,6 & 1,2 & 1,2 \\
\hline Dette totate " (mililiards \$) & 49,0 & 50,0 & 38,8 & 39,8 & 41,0 & 42,2 & 43,7 & 45,5 & 47,9 & 50,1 & 52,5 & 54,9 \\
\hline IdempiB & 127,9 & 133,0 & 126,6 & 141,4 & 142,7 & 142,1 & 139,1 & 134,0 & 130,0 & 125,7 & 122,6 & $\$ 19,2$ \\
\hline Service de la delle (militiars \$) & 5,1 & 6,3 & 5,9 & 5,6 & 5,4 & 5,7 & 5.8 & 6,1 & 5,9 & 6,1 & 6,3 & 7,0 \\
\hline Ide,Jkp. de biens et services " & 42,2 & 47,6 & 43,5 & 39,6 & 35,4 & 34,4 & 33,0 & 33,0 & 29.7 & 27,9 & 26,8 & 27,8 \\
\hline Investissements:PlB & 23,6 & 23,1 & 19,0 & 16,0 & 18,0 & 21,0 & 23,0 & 24,0 & 24,5 & 25,0 & 25,0 & 25,0 \\
\hline Epargne/PlB & 10,2 & 11,0 & 6,2 & 4,1 & 7,4 & 10,5 & 121 & 13,0 & 13,3 & 14,3 & 14,7 & 15,1 \\
\hline Déficit budgétaire/Plg & $\cdot 18,3$ & $\cdot 18,4$ & $-21,9$ & $.10,2$ & $-6,6$ & $.5,4$ & $\cdot 3,3$ & $\cdot 1,5$ & - & - & - & - \\
\hline $\begin{array}{l}\text { Croissance des exportations de } \\
\text { biens et sevices ron factiurs }\end{array}$ & & & & & & & & & & 5 & 40 & 50 \\
\hline ExportalionsSPIB & 18,9 & 20,1 & 20,0 & 21,7 & 23,3 & $\begin{array}{l}24,2 \\
24,2\end{array}$ & 24,3 & 24,2 & 24,0 & 24,4 & 24,6 & 24,8 \\
\hline Croissance des importations de & & & & & & & & & & & & \\
\hline biens et sonvices non lacleurs & $-4,1$ & 2,3 & 3,3 & $.5,4$ & 1,4 & 3.8 & 4,0 & 4,1 & 3,7 & 3.4 & 3,2 & 3.2 \\
\hline Importationsipla & 32,30 & 32,6 & 34,2 & 33,4 & 33,3 & 33,6 & 34,0 & 34,0 & 33,9 & 33,7 & 33,4 & 33,2 \\
\hline
\end{tabular}

- Ne prend pas en compte raccord du Cletb de Paris de juillet 1991. En cas contraire, les indicateurs ci-dessus pour l'an 2000 setaient 31,4-68,4-3,6-14,5. $\because$ N.8. 1989 signilie année financière 1988-89, et ainsi de suile.

Source : Banque Mondiale, juin 1991. 
Tableau 6 : Évolution de la balance des paiements (millions \$)

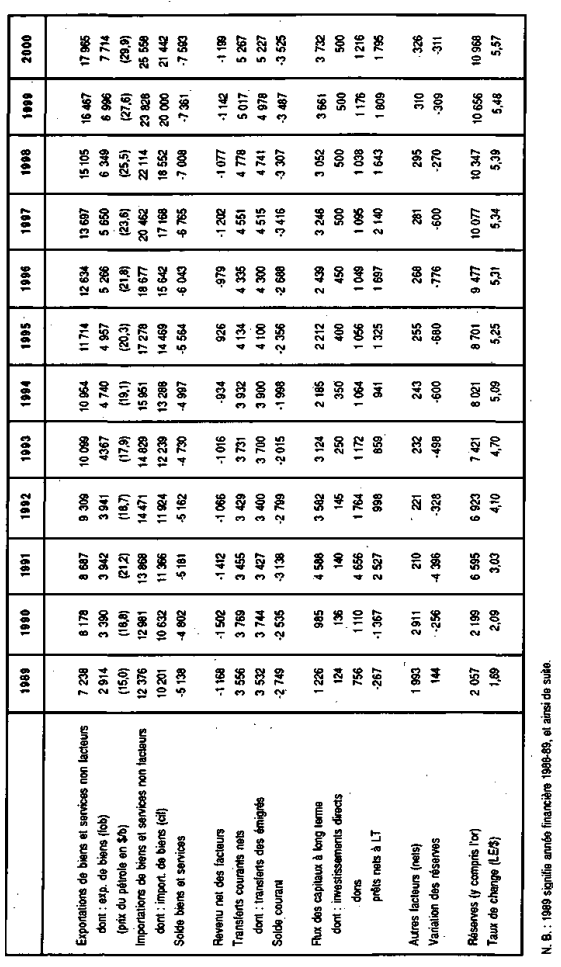

Source : Banque Mondiale, juin 1991

141 Le financement extérieur de l'économie devrait rester important, avec 7,5 milliards \$ de dons et 8,8 milliards \$ de prêts à long terme de 1990-91 à 1992-93 (compte tenu des remises de dettes), soit 5,4 milliards \$ d'aide internationale par an. Les investissements directs devraient légèrement augmenter, à 500 millions $\$ / a n$.

\section{Conclusion}

142 «C'est la première fois que l'Égypte met en oeuvre un programme de réformes en respectant les délais », indiquait $\mathrm{M}$. Abdel Chakour Chaalan, directeur du département Moyen-Orient au FMI, après que le Fonds a décidé de débloquer la deuxième tranche de son crédit de confirmation ${ }^{11}$. Les résultats du programme de stabilisation forcent en effet le respect des sceptiques, qui étaient les plus nombreux à l'origine. Le plus spectaculaire a été l'excédent inattendu de la balance des paiements en 1991, estimé à 1 milliard \$, alors qu'un déficit de 2,5 milliards \$ était prévu. Les experts s'attendaient également à une dépréciation de la livre plus importante et à une inflation plus forte (elle ne dépassait pas $26 \%$ en glissement annuel en janvier 1992, dernier chiffre connu).

Si la politique monétaire reçoit un satisfecit, il n'en va pas de même pour certaines réformes de structure préconisées par le programme d'ajustement, qui posent de délicats problèmes sociopolitiques. Il est par ailleurs possible que les autorités égyptiennes aient jugé préférable de mécontenter la Banque Mondiale plutôt que le FMI, compte tenu de leur poids respectif. L'arrivée au Caire en janvier 1992 du premier représentant de la Banque en Égypte s'inscrit dans ce contexte. 
144 M. Caio Koch-Weser, vice-président de la Banque Mondiale pour le Moyen-Orient et l'Afrique du Nord, ne pouvait alors qu'avouer, à l'issue de sa première visite au Caire, que « la réponse de l'économie égyptienne avait été meilleure qu'escomptée il y a un an $»^{12}$ mais il ajoutait que « la restructuration du secteur public avait été plus lente que prévue » et mentionnait comme deuxième différend l'inaction du Fonds social.

Quant à M. Spiros Voyadzis, chef de la division de la Banque chargé de superviser les prêts à l'Égypte, il estimait :

«En ce qui concerne la stabilisation, les résultats ont largement dépassé nos espérances. Nous nous attendons, pour 1992, à un rééquilibrage plus important que prévu de la balance des paiements, lié essentiellement à la réduction de la dette consentie par les créanciers du Club de Paris et à une hausse récente des revenus en devises provenant du tourisme, du rapatriement des salaires des travailleurs émigrés, des apports de capitaux et des recettes du canal de Suez. En outre, les réserves internationales de l'Égypte sont passées de quelque 3 milliards \$ il y a environ deux ans à plus de 8 milliards \$ à la moitié de l'année fiscale en cours. Alors que les premières estimations faisaient état d'une baisse du PIB, le pays devait en fait enregistrer une légère croissance économique cette année. En ce qui concerne l'ajustement structurel, il est vrai que certaines opérations, notamment les privatisations et les réformes des entreprises publiques, évoluent très lentement. Toutefois, les autres actions du programme d'ajustement sont en bonne voie: réformes commerciales, libéralisation des prix et réduction du contrôle de la production et de l'investissement. $»^{13}$

Si la période critique de la stabilisation peut s'avérer relativement courte, l'ajustement structurel est quant à lui une oeuvre de longue haleine. C'est pourquoi la Banque Mondiale a d'ores et déjà commencé à préparer sa seconde phase, qu'elle envisage de financer par un deuxième prêt à l'ajustement structurel. Les principales mesures prévues seraient la diversification des instruments de la politique monétaire, la réforme de la fiscalité sur les revenus et les entreprises, la création de nouveaux produits d'épargne, la mobilisation de ressources en devises diversifiées, la réforme bancaire et des réformes du système des assurances, de la sécurité sociale et des fonds de retraite.

147 On constate donc que certains secteurs, notamment celui de la défense, continuent à échapper aux négociations sur l'ajustement structurel, bien qu'ils soient indirectement touchés.

Les institutions financières internationales, les autres bailleurs de fonds et les autorités égyptiennes se retrouvent pour juger en définitive que l'Égypte a réussi un travail ardu jusqu'à présent, mais qu'il lui reste la partie la plus difficile à accomplir.

\section{NOTES}

1. L'année fiscale égyptienne va du $1^{\mathrm{er}}$ juillet au 30 juin.

2. «Pour éviter d'avoir à batailler avec les groupes de pression professionnels, politiques ou syndicaux, les gouvernements cachent leurs véritables intentions. [...] Ceci met clairement en évidence le décalage qui existe entre le discours officiel, c'est-à-dire le discours politique, et la 
réalité ", écrit Alain Vemholes à propos... du projet de loi de finances pour 1992 du gouvernement français (Le Monde, 11 décembre 1991, p. 21). On voit qu'aucun pays n'a l'exclusivité de ce genre de pratiques.

3. Le jeu de mots est particulièrement réussi en arabe, puisque le Fonds Monétaire International (Sunduq an-Naqd ad-Dawli) devient par un seul léger changement de consonne Fonds de la Misère Internationale (Sunduq an-Nakd ad-Dawli).

4. Voir Égypte/Monde Arabe (EMA), 8, $4^{\mathrm{e}}$ trimestre 1991, p. 142.

5. EMA 7, $3^{\mathrm{e}}$ trimestre 1991, p. 237.

6. Pour un exposé détaillé de cette loi, voir mon article sur la réforme des entreprises publiques dans EMA 7, 3 $3^{\text {e }}$ trimestre 1991, p. 252-255.

7. «Les gouvernements des pays démocratiques manquent-ils à ce point d'autorité pour obtenir des Parlements le vote de réformes difficiles ou impopulaires?» (Le Monde, art. cité).

8. Cf. détails de la politique égyptienne de privatisation dans mon article d'EMA 8, $4^{\mathrm{e}}$ trimestre 1991, p. 166-172.

9. Voir EMA 6, $2^{\mathrm{e}}$ trimestre 1991, p. 263 et 7, $3^{\mathrm{e}}$ trimestre 1991, p. 250.

10. Détails dans EMA 8, 4e trimestre 1991, p. 146.

11. Middle East Economic Digest, 6 décembre 1991.

12. Entretien du 29 janvier 1992.

13. Banque Mondiale Actualités, mars 1992.

\section{INDEX}

Mots-clés : ajustement structurel, économie, politique économique

\section{AUTEUR}

LOUIS BLIN

Cedej 